\title{
Fractional dynamics in the Rayleigh's piston
}

\author{
J.A. Tenreiro Machado
}

\author{
A B S T R A C T
}

This paper studies the dynamics of the Rayleigh piston using the modeling tools of Fractional Calculus. Several numerical experiments examine the effect of distinct values of the parameters. The time responses are transformed into the Fourier domain and approximated by means of power law approximations. The description reveals characteristics usual in Fractional Brownian phenomena.

Keywords:

Thermodynamics

System modelling

Fractional calculus

Fractional Brownian motion

Power law

\section{Introduction}

During the last decades several papers addressed a conceptual example of statistical mechanics known as the "Rayleigh piston" $[1,2]$. This classical prototype system consists of a one-dimensional array of particles separated by means of an adiabatic piston. The particles in the two cylinders have non-zero random velocities and collide sporadically with the piston provoking its motion. While a very simple system, a kind of conceptual paradox occurs and considerable debate took place about the steady state operating conditions. Nevertheless, most of the technical literature addresses the relationship of the system final equilibrium conditions and the study of the complex dynamics has not attracted relevant attention.

This paper focus the dynamics of the Rayleigh piston in the perspective of Fractional Brownian motion (fBm) and Fractional Calculus (FC). The fBm was introduced by Kolmogorov [3]. Later Mandelbrot adopted the concept of fBm to model phenomena with self-similarity and long range effects [4]. The fBm is also called $1 / f$ noise [5], where $f$ denotes frequency, because its spectrum is given by $1 / f^{\alpha}, \alpha>0$. The fBm is interpreted as a signature of complexity [6] and has been observed in many distinct areas [7], namely in economics and finance [8,9], geophysics [10-15], music and speech [16-18], biology [19-23] and others. During the last years the relation between fBm and FC was studied by some researchers [24-27]. FC emerged with the ideas of Leibniz and several important mathematicians contributed to its development [28-32]. However, only in the last decades [33,34] FC was recognized to be an important tool to study systems with long range memory phenomena [35-44]. FC generalizes the operations of integration and differentiation to non-integer orders and constitutes an efficient mathematical tool for describing natural phenomena with long-range memory effects and power law description. This paper addresses the Rayleigh piston and its characterization by means of fBm and FC concepts.

Having these ideas in mind, this paper focus on the fBm in the perspective of FC and is organized as follows. Section 2 introduces the "Rayleigh piston", develops the analysis in the Fourier domain, extracting several power-law parameters, and discusses the results in the perspective of dynamical systems. Finally, Section 3 outlines the main conclusions. 


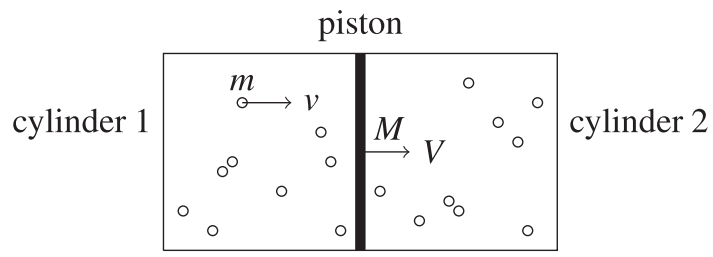

Fig. 1. The Rayleigh piston.

\section{Preliminary concepts}

The Rayleigh's piston is a system consisting of two cylinders, to be denoted as 1 and 2, containing some type of fluid, and separated by an adiabatic movable piston (Fig. 1). A brake maintains the piston at rest until time $t=0$. The two fluids are in equilibrium with pressure, volume and temperature $\left\{p_{i}(0), V_{i}(0), T_{i}(0)\right\}, i=1,2$. The piston with mass $M$ undergoes random one-dimensional collisions with particles of mass $m$. Furthermore, there are $n_{i}, i=1,2$, particles per unit volume, with Maxwell distributed velocities at temperature $T_{i}$.

In steady state occurs a mechanical equilibrium and the pressures are identical, that is, $p_{1}(t \rightarrow \infty)=p_{2}(t \rightarrow \infty)$. However, nothing can be said about the final temperatures $T_{1}(t \rightarrow \infty)$ and $T_{2}(t \rightarrow \infty)$, since the laws of thermostatics are insufficient to predict them. The reader can follow the discussion about this gedankenexperiment in [45-54] and references therein.

In this paper we focus the dynamics of the motion of the piston for different operating conditions under the light of FC. At $t=0$ the particles of cylinder $i, i=1,2$, are considered to have a one-dimensional probability distribution so that $v_{i} \sim e^{-\frac{v_{i}^{2}}{\sigma_{i}}}$. The collision phenomenon is modeled by means of the pair of initial and final velocities, $\left(V_{i}, v_{i}\right)$ and $\left(V_{f}, v_{f}\right)$, respectively. Elastic collisions satisfy the conservation of energy and momentum:

$$
\begin{aligned}
& E_{f}+e_{f}=E_{i}+e_{i}, \\
& P_{f}+p_{f}=P_{i}+p_{i},
\end{aligned}
$$

where subscripts $i$ and $f$ denote the initial and final states, $P=M V$ and $p=m v$ denote momenta and $E=\frac{1}{2} M V^{2}$ and $e=\frac{1}{2} m v^{2}$ the kinetic energies, of the piston and particles, respectively. Therefore, the velocities of the piston and the particle after collision, are given by:

$$
\begin{aligned}
& V_{f}=V_{i}-\frac{2 m}{m+M}\left(V_{i}-v_{i}\right), \\
& v_{f}=v_{i}-\frac{2 M}{m+M}\left(v_{i}-V_{i}\right) .
\end{aligned}
$$

During the following numerical simulations we adopt a time step of $h=0.5 \cdot 10^{-3}$, an initial piston position $x(0)=0$, and two identical cylinders with unit width.
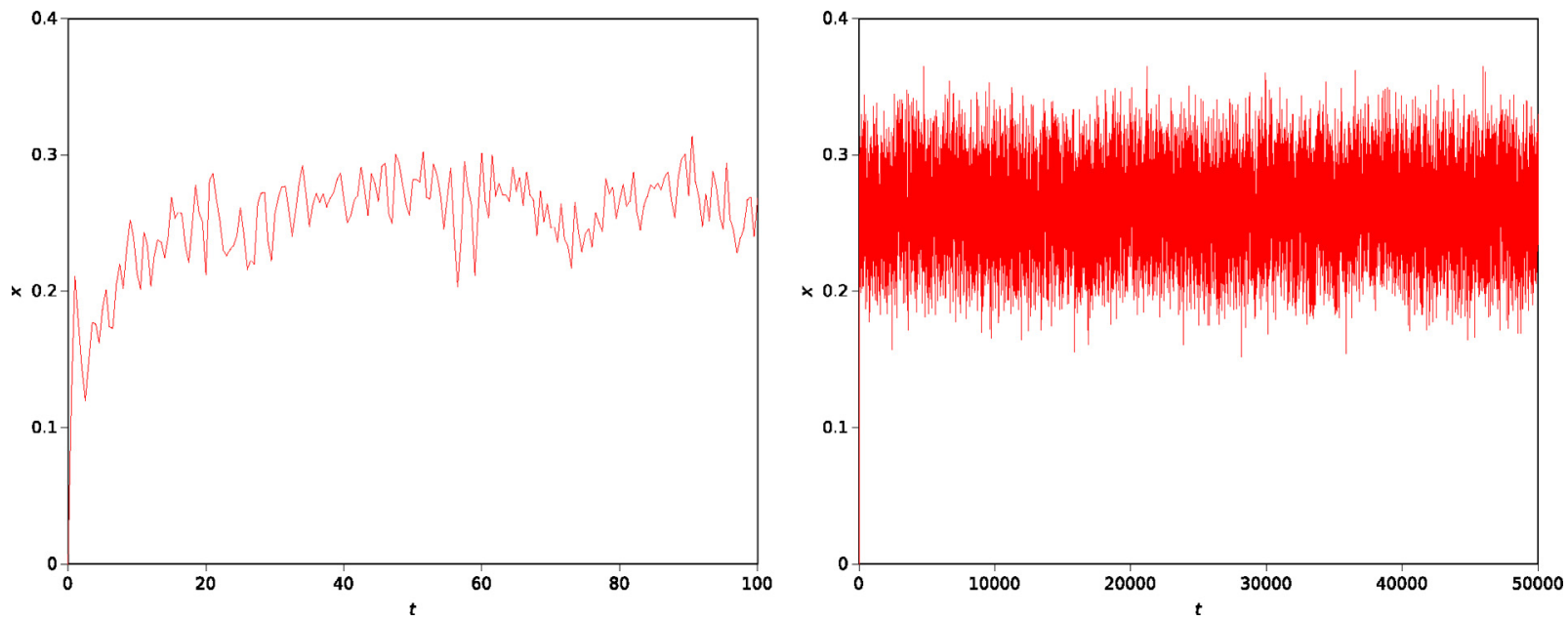

Fig. 2. Time response $x(t)$ of the piston position for $0 \leq t \leq 100$ (left) and $0 \leq t \leq 5 \cdot 10^{4}$ (right), with $M=10, m=1, n_{1}=1000, n_{2}=500, \sigma_{1}=\sigma_{2}=1.0$, $h=0.5 \cdot 10^{-3}$. 

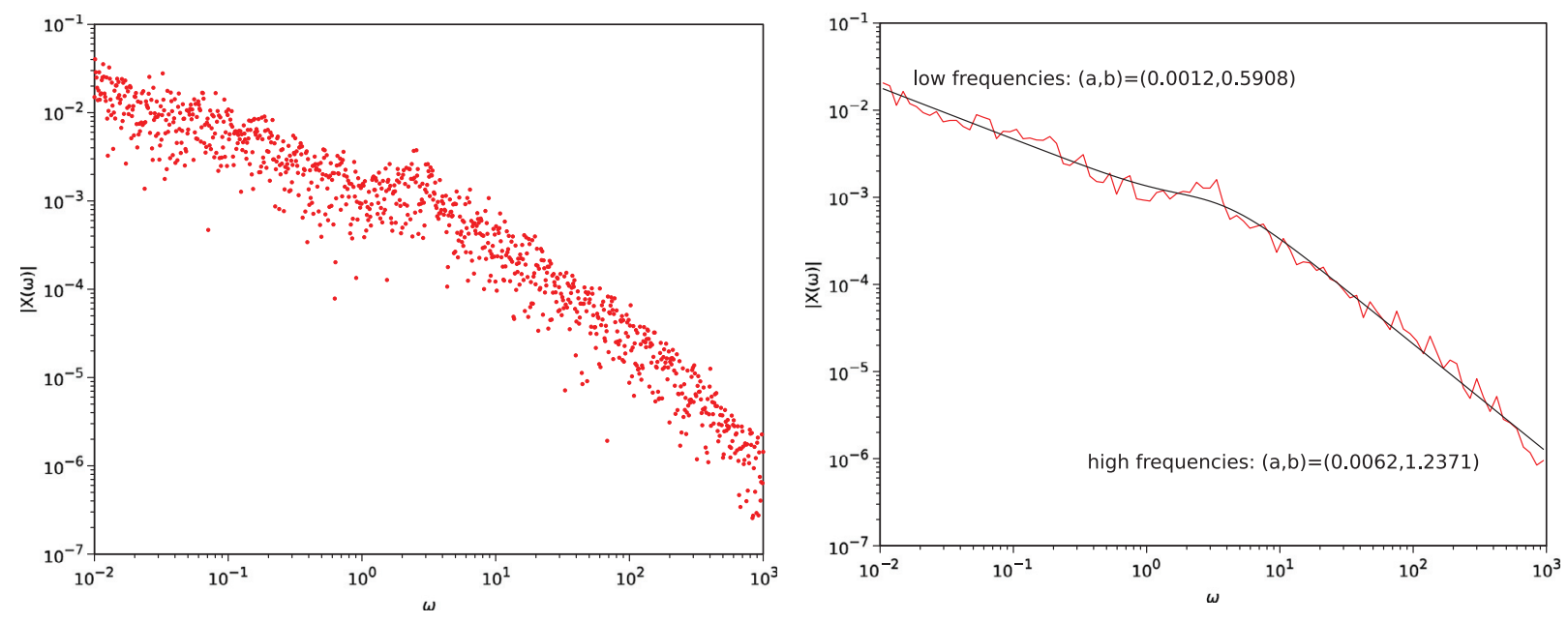

Fig. 3. Amplitude of the frequency response $|X(\omega)|$ versus $\omega$ of the piston position with $M=10, m=1, n_{1}=1000, n_{2}=500, \sigma_{1}=\sigma_{2}=1.0,0 \leq t \leq 5 \cdot 10^{4}$, $h=0.5 \cdot 10^{-3}$. Left: initial response, right: moving average and approximations $(4)$ leading to the PL $\left(a_{l}, b_{l}\right)=(0.0012,0.5908)$ and $\left(a_{h}, b_{h}\right)=(0.0062,1.2371)$.

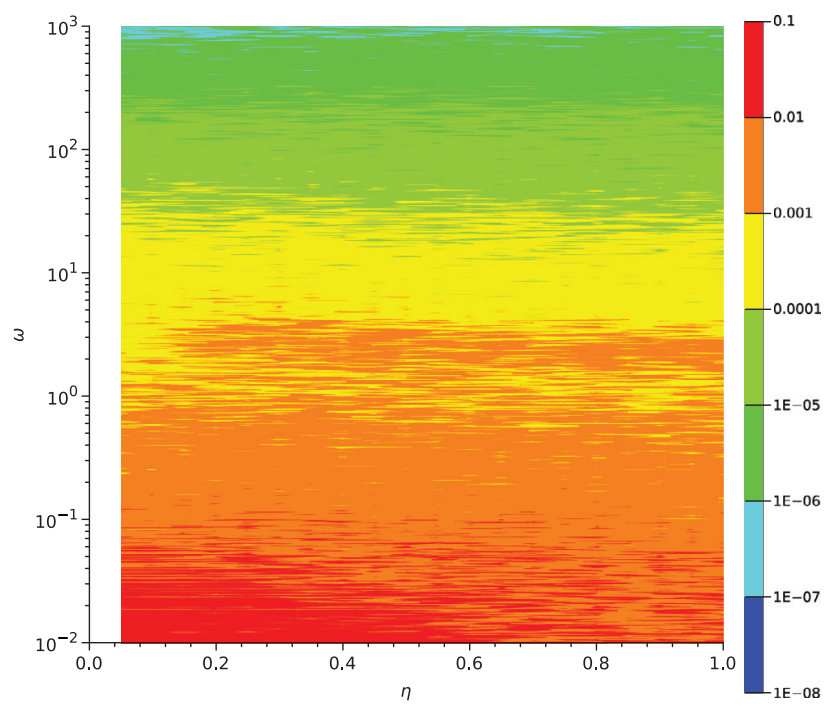

Fig. 4. Amplitude of the frequency response $|X(\omega)|$ versus $(\omega, \eta)$ of the piston position with $M=10, m=1, \sigma_{1}=\sigma_{2}=1.0,0 \leq t \leq 5 \cdot 10^{4}, h=0.5 \cdot 10^{-3}$.
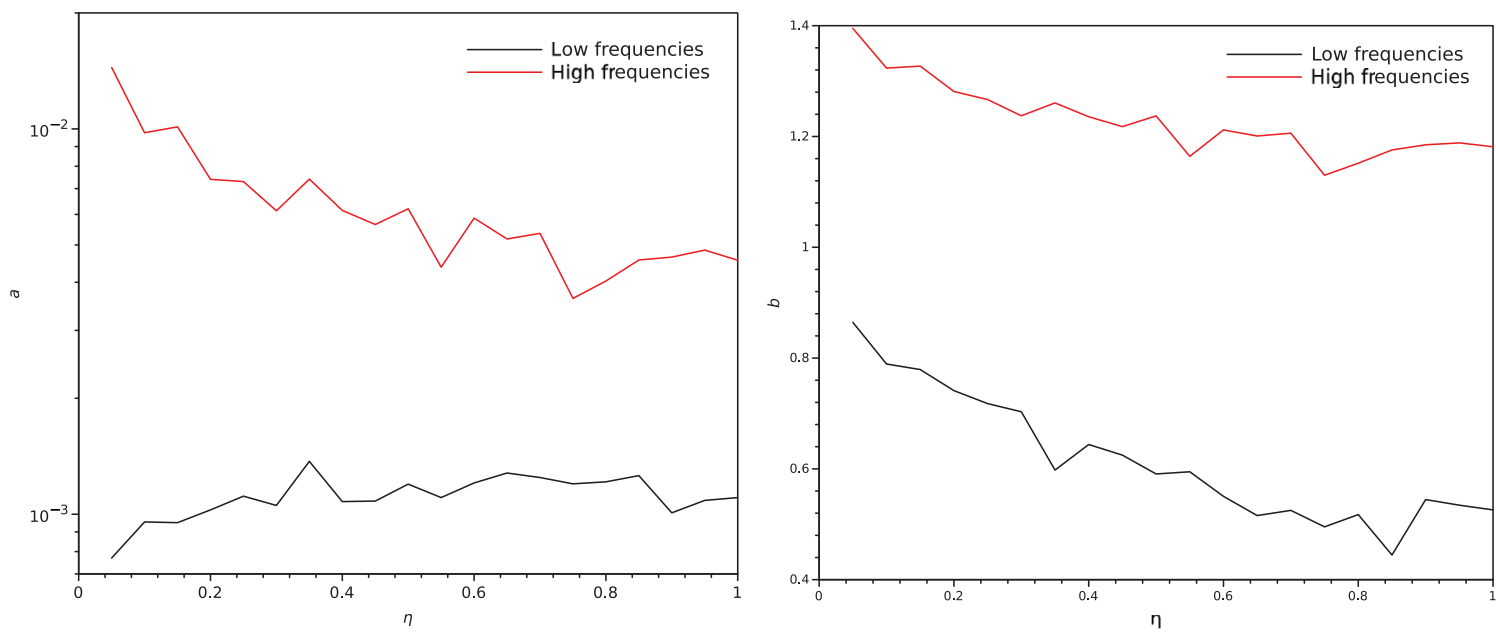

Fig. 5. Variation of parameters $a_{l}$ and $a_{h}$, and $b_{l}$ and $b_{h}$, versus $\eta=\frac{n_{2}}{n_{1}}$, for $0 \leq t \leq 5 \cdot 10^{4}$, with $M=10, m=1, \sigma_{1}=\sigma_{2}=1.0$. 
Fig. 2 shows the transient (left) and the long term behavior (right) of the piston position time response $x(t)=\int_{0}^{t} v(\tau) d \tau$, respectively, for $M=10, m=1, n_{1}=1000, n_{2}=500, \sigma_{1}=\sigma_{2}=1.0$. In the transient we observe a fast initial evolution followed by a much slower response. In what concerns the steady-state response we verify that the long term behavior reveals fBm representative of the particles' dynamical effects.

Fig. 3 depicts the amplitude of the corresponding Fourier spectrum, $|\mathcal{F}\{x(t)\}|=|X(\omega)|$. At the left is represented the frequency response for 1000 points and at the right is shown a 10 point moving average and the approximation

$$
|X(\omega)|=\left|K \frac{\left(1+\frac{s}{z}\right)^{\beta}}{s^{\alpha_{1}}\left(1+\frac{s}{p}\right)^{\alpha_{2}}}\right|_{s=j \omega}, K, z, p, \alpha_{1}, \alpha_{2}, \beta \in \mathbb{R}^{+} .
$$

The experiments demonstrated that the values of $z, p$ and $\beta$ are of minor importance, being relevant the power law (PL) approximations at low and high frequencies of the type $|X(\omega)| \approx a \omega^{-b}, a, b \in \mathbb{R}^{+}$. Therefore, in the sequel we focus the attention in the low and high PL trendlines:

$$
\begin{aligned}
& |X(\omega)| \approx a_{l} \omega^{-b_{l}}=K \cdot s^{-\alpha_{1}}, a_{l}, b_{l} \in \mathbb{R}^{+}, \\
& |X(\omega)| \approx a_{h} \omega^{-b_{h}}=K \frac{p^{\alpha_{2}}}{z^{\beta}} \cdot s^{-\left(\alpha_{1}+\alpha_{2}-\beta\right)}, a_{h}, b_{h} \in \mathbb{R}^{+},
\end{aligned}
$$

at the low and high frequency ranges, respectively.

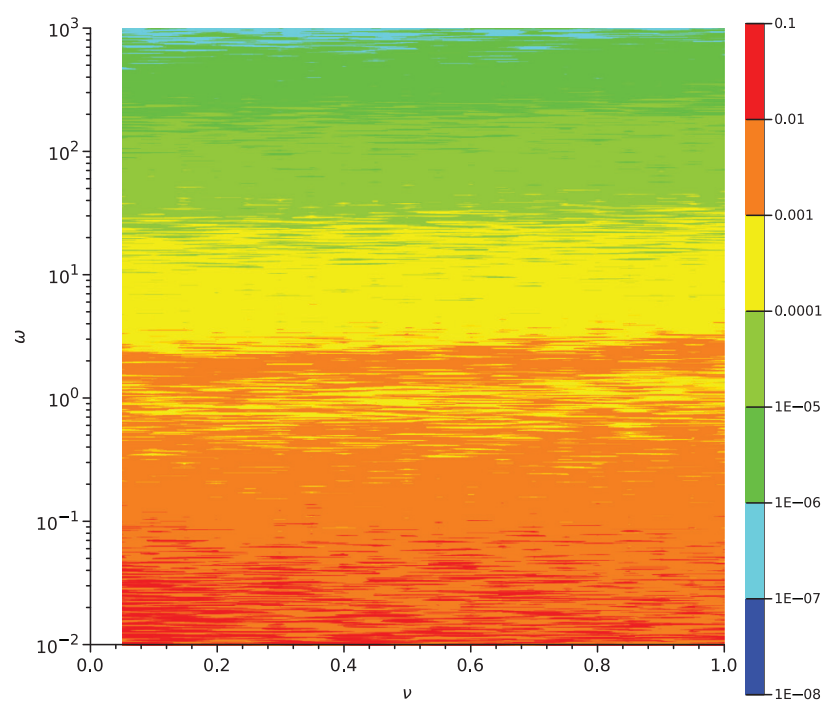

Fig. 6. Amplitude of the frequency response $|X(\omega)|$ versus $(\omega, v)$ of the piston position with $M=10, m=1, n_{1}=n_{2}=1000, \sigma_{1}=\sigma_{2}=1.0,0 \leq t \leq 5 \cdot 10^{4}$, $h=0.5 \cdot 10^{-3}$.
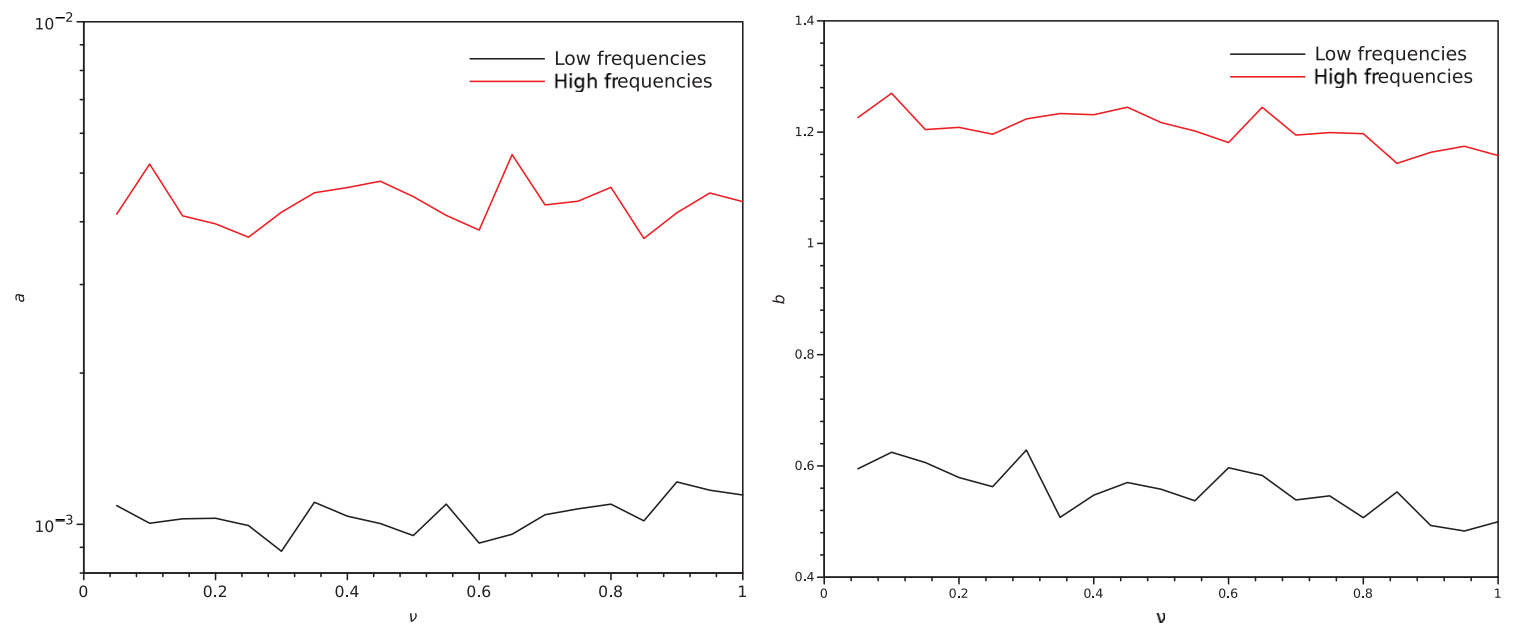

Fig. 7. Variation of parameters $a_{l}$ and $a_{h}$, and $b_{l}$ and $b_{h}$, versus $v=\frac{\sigma_{2}}{\sigma_{1}}$ of the piston position with $M=10, m=1, n_{1}=n_{2}=1000,0 \leq t \leq 5 \cdot 10^{4}, h=0.5 \cdot 10^{-3}$. 


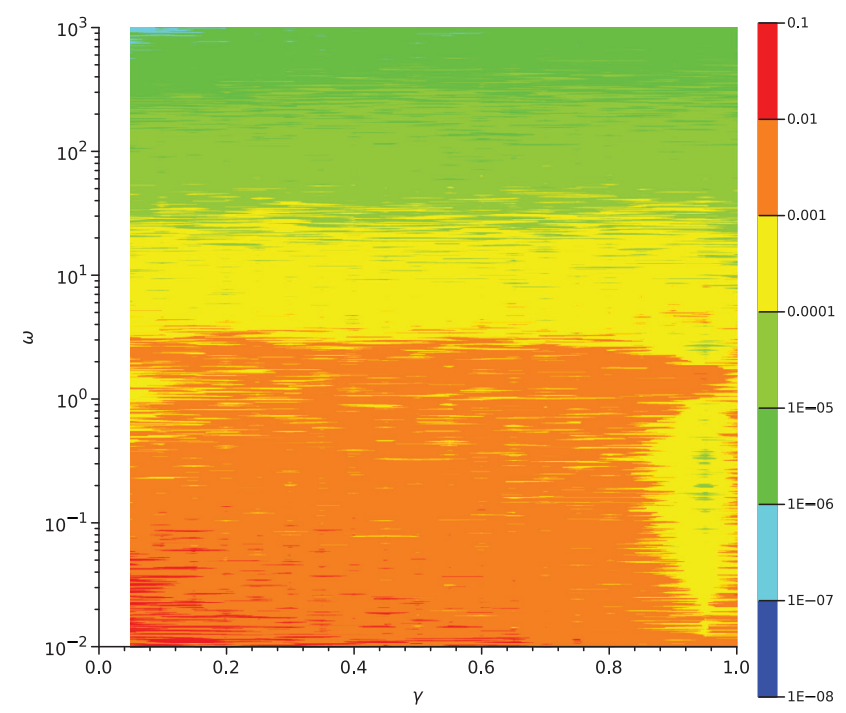

Fig. 8. Amplitude of the frequency response $|X(\omega)|$ versus $(\omega, \gamma)$ of the piston position with $n_{1}=n_{2}=1000, \sigma_{1}=\sigma_{2}=1.0,0 \leq t \leq 5 \cdot 10^{4}, h=0.5 \cdot 10^{-3}$.
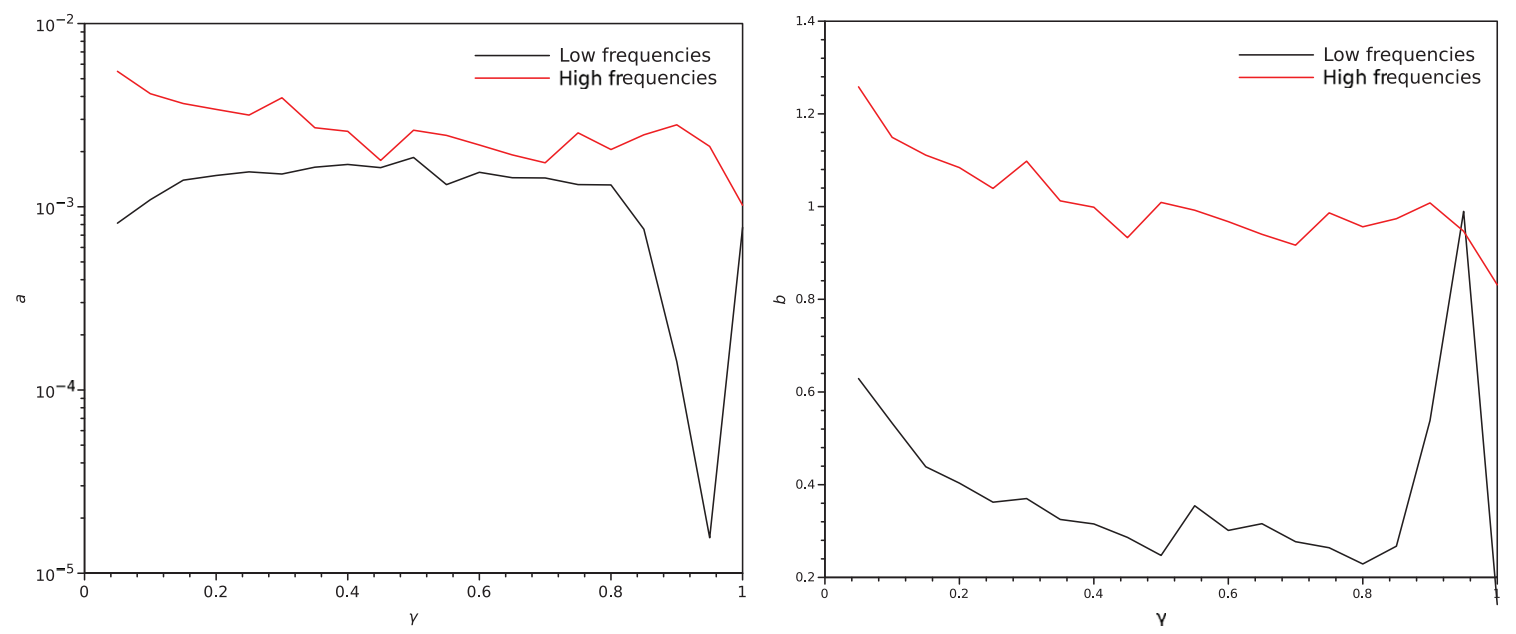

Fig. 9. Variation of parameters $a_{l}$ and $a_{h}$, and $b_{l}$ and $b_{h}$, versus $\gamma=\frac{m}{M}$, for $0 \leq t \leq 5 \cdot 10^{4}$, with $n_{1}=n_{2}=5000, \sigma_{1}=\sigma_{2}=1.0$.

We verify that $|\mathcal{F}\{x(t)\}|$ has distinct characteristics, but fractional in both cases.

Having these charts in mind, in a first set of experiments we vary $\eta=\frac{n_{2}}{n_{1}}$ while keeping constant $\nu=\frac{\sigma_{2}}{\sigma_{1}}$ and $\gamma=\frac{m}{M}$. Figs. 4 and 5 depict the locus $|X(\omega)|$ versus $(\omega, v)$ and the variation of parameters $a$ and $b$ (i.e., the pairs $a_{l}, a_{h}$, and $\left.b_{l}, b_{h}\right)$ versus $\eta$, respectively. Besides some "noise" in the charts, due to numerical approximations and the stochastic nature of the collision, we verify that at $b_{l}$ and $b_{h}$ are significantly distinct for low values of $\eta$, but tend to stabilize as $\eta \rightarrow 1$.

In a second set of experiments we keep constant $\eta$ and $\gamma$ while varying $\nu$. Figs. 6 and 7 depict the locus $|X(\omega)|$ versus $(\omega, v)$ and the variation of the pairs $a_{l}, a_{h}$, and $b_{l}, b_{h}$, versus $v$, respectively. The parameters exhibit a much smaller variation than in the previous case.

In a third set of experiments we keep constant $\eta$ and $v$ while varying $\gamma$. Figs. 8 and 9 depict the locus $|X(\omega)|$ versus $(\omega$, $v$ ) and the variation of the pairs $a_{l}, a_{h}$, and $b_{l}, b_{h}$, versus $\gamma$, respectively. We note an interesting phenomenon for $0.8<\gamma<$ 0.95 with a kind of peak in the frequencies as shown in Fig. 10 (for $\gamma=0.9$ ) with a considerable variation of the parameters $a_{l}$ and $b_{l}$.

In all cases was verified the existence of $\mathrm{fBm}$, the presence of fractional order dynamics characterized by means of power law spectra, and the co-existence of distinct behaviors in the transient and steady-state time responses, that is to say, in the low and high frequencies. 

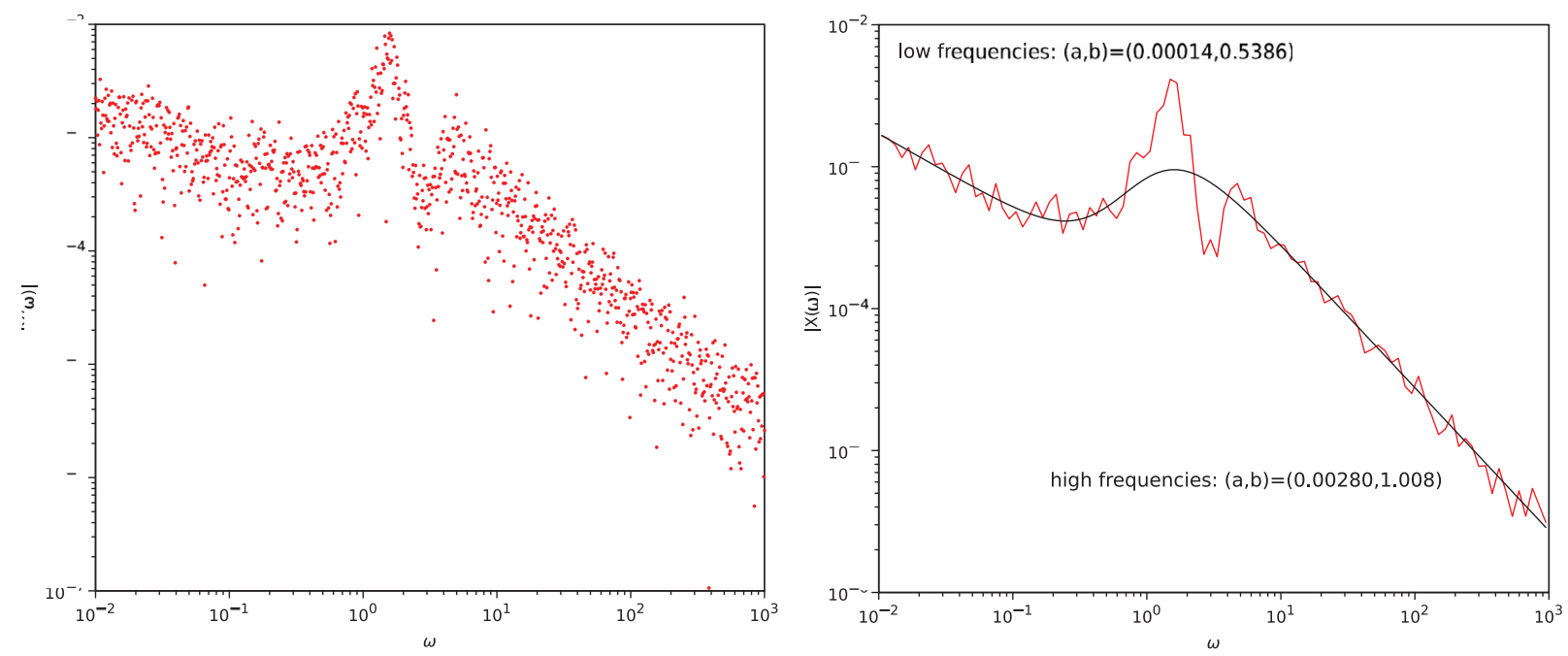

Fig. 10. Amplitude of the frequency response $|X(\omega)|$ versus $\omega$ of the piston position with $M=10, m=1, n_{1}=1000, n_{2}=500, \gamma=0.9,0 \leq t \leq 5 \cdot 10^{4}, h=$ $0.5 \cdot 10^{-3}$. Left: initial response, right: moving average and PL approximations $\left(a_{l}, b_{l}\right)=(0.0012,0.5908)$ and $\left(a_{h}, b_{h}\right)=(0.0062,1.2371)$.

\section{Conclusions}

This paper studied the dynamical properties of Rayleigh piston. The novel contribution was in the viewpoint of fBm and FC. Several numerical experiments with distinct values for the system parameters, such as number of particles, their masses and their velocities, were conducted. The transient and steady-state behavior was characterized in the Fourier domain by means of power law approximations. The results demonstrated that fBm and FC are useful tools for investigating the complexity present in this classical system.

\section{References}

[1] Callen HB. Thermodynamics. New York: John Wiley and sons; 1963.

[2] Callen HB. Thermodynamics and an introduction to thermostatistics. 2nd ed. New York: John Wiley and sons; 1985.

[3] Kolmogorov AN. Wienersche spiralen und einige andere interessante kurven im hilbertschen raum. Comptes Rendus (Doklady) Acad Sci l'URSS 1940;26(2):115-18.

[4] Mandelbrot BB, Ness JWV. The fractional Brownian motions, fractional noises and applications. SIAM Rev 1968;10(4):422-37. doi:10.1137/1010093.

[5] Keshner MS. 1/f noise. Proc IEEE 1982;70(3):212-18. doi:10.1109/PROC.1982.12282.

[6] Mandelbrot BB. The fractal geometry of nature. New York: W. H. Freeman; 1983.

[7] Nourdin I. Selected aspects of fractional Brownian motion. Milan: Springer; 2012

[8] Lo AW. Long term memory in stock market prices. Econometrica 1991;59(5):1279-313. doi:10.2307/2938368

[9] Baillie RT. Long memory processes and fractional integration in econometrics. J Econom 1996;73(1):5-59. doi:10.1016/0304-4076(95)01732-1.

[10] Király A, Jánosi IM. Stochastic modeling of daily temperature fluctuations. Phys Rev E 1998;65(051102):1-6. http://dx.doi.org/10.1103/PhysRevE.65.051102.

[11] Montanari A, Rosso R, Taqqu MS. A seasonal fractional ARIMA model applied to the Nile river monthly flows at Aswan. Water Resour Res 2000;36(5):124959. doi:10.1029/2000WR900012.

[12] Koutsoyiannis D. Climate change, the Hurst phenomenon, and hydrological statistics. Hydrol Sci J 2003;48(1):3-24. doi:10.1623/hysj.48.1.3.43481.

[13] Li C-F. Rescaled-range and power spectrum analyses on well-logging data. Geophys J Int 2003;153(1):201-12. doi:10.1046/j.1365-246X.2003.01893.X.

[14] chih Chen C, Lee Y-T, Chang Y-F. A relationship between Hurst exponents of slip and waiting time data of earthquakes. Physica A 2008;387(18):4643-8. doi:10.1016/j.physa.2007.08.063.

[15] Vyushin DI, Kushner PJ, Mayer J. On the origins of temporal power-law behavior in the global atmospheric circulation. Geophys Res Lett 2009;36(14):L14706. doi:10.1029/2009GL038771.

[16] Voss RF, Clarke J. 1/f noise in music and speech. Nature 1975;258:317-18. doi:10.1038/258317a0

[17] Voss RF, Clarke J. "1/f noise" in music: music from 1/f noise. J Acoust Soc Am 1978;63(1):258-63. http://dx.doi.org/10.1121/1.381721.

[18] Ro W, Kwon Y. 1/f noise analysis of songs in various genre of music. Chaos Solitons Fractals 2009;42(4):2305-11. doi:10.1016/j.chaos.2009.03.129.

[19] Peng CK, Buldyrev S, Goldberger A, Havlin S, Sciortino F, Simons M, Stanley HE. Long-range correlations in nucleotide sequences. Nature 1992;356:168-71. doi:10.1038/356168a0.

[20] Peng C-K, Buldyrev S, Havlin S, Simmons M, Stanley H, Goldberger A. Mosaic organization of DNA nucleotides. Phys Rev E 1994;49(2):1685-9. http://dx.doi.org/10.1103/PhysRevE.49.1685.

[21] Voss RF. Evolution of long-range fractal correlation and $1 / f$ noise in DNA base sequences. Phys Rev Lett 1992;68:3805-8. http://dx.doi.org/10.1103/PhysRevLett.68.3805.

[22] Som A, Chattopadhyay S, Chakraborth J, Bandyopadhyay D. Codon distribution in DNA, physical review E: statistica. Nonlinear Soft Matter Phys 2001;63(5). http://dx.doi.org/10.1103/PhysRevE.63.051908.

[23] Kim J, Kwon Y, Yang S, Lee E. Codon and amino-acid distribution in DNA. Chaos Solitons Fractals 2005;23(5):1795-807. doi:10.1016/j.chaos.2004.07.027.

[24] Ortigueira MD, Batista AG. A fractional linear system view of the fractional Brownian motion. Nonlinear Dyn 2004;38(1-4):295-303. doi:10.1007/s11071$004-3762-8$

[25] Ortigueira MD, Batista AG. On the relation between the fractional Brownian motion and the fractional derivatives. Phys Lett A 2008;372(7):958-68. doi:10.1016/j.physleta.2007.08.062.

[26] Deng W, Barkai E. Ergodic properties of fractional Brownian-Langevin motion. Phys Rev E: Stat Nonlinear Soft Matter Phys 2009;79(1). http://dx.doi.org/10.1103/PhysRevE.79.011112. 
[27] Grigolini P, Aquino G, Bologna M, Luković M, West BJ. A theory of 1/f noise in human cognition. Phys A: Stat Mech Appl 2009;388(19):4192-204. doi:10.1016/j.physa.2009.06.024.

[28] Oldham K, Spanier J. The fractional calculus: theory and application of differentiation and integration to arbitrary order. New York: Academic Press; 1974.

[29] Samko S, Kilbas A, Marichev O. Fractional integrals and derivatives: theory and applications. Amsterdam: Gordon and Breach Science Publishers; 1993.

[30] Miller K, Ross B. An introduction to the fractional calculus and fractional differential equations. New York: John Wiley and Sons; 1993.

[31] Podlubny I. Fractional differential equations. An introduction to fractional derivatives fractional differential equations, to methods of their solution, mathematics in science and engineering, vol. 198. San Diego: Academic Press; 1998.

[32] Kilbas A, Srivastava H, Trujillo J. Theory and applications of fractional differential equations. North-Holland Mathematics Studies, vol. 204. Amsterdam: Elsevier; 2006.

[33] Machado JT, Kiryakova V, Mainardi F. Recent history of fractional calculus. Commun Nonlinear Sci Numer Simul 2011;16(3):1140-53. doi:10.1016/j.cnsns.2010.05.027.

[34] Machado JT, Galhano AM, Trujillo JJ. On development of fractional calculus during the last fifty years. Scientometrics 2014;98(1):577-82. doi:10.1007/s11192013-1032-6.

[35] Ortigueira MD, Machado JAT. Fractional signal processing and applications. Signal Process 2003;83(11):2285-6. doi:10.1016/S0165-1684(03)00181-6.

[36] Ortigueira MD, Machado JAT. Fractional calculus applications in signals and systems. Signal Process 2006;86(10):2503-4. doi:10.1016/j.sigpro.2006.02.001.

[37] Sabatier J, Agrawal OP, Machado JT. Advances in fractional calculus: theoretical developments and applications in physics and engineering. Dordrecht, The Netherlands: Springer; 2007.

[38] Ionescu C, Keyser RD. Relations between fractional-order model parameters and lung pathology in chronic obstructive pulmonary disease. IEEE Trans Biomed Eng 2009;56(4):978-87. doi:10.1109/TBME.2008.2004966.

[39] Mainardi F. Fractional calculus and waves in linear viscoelasticity: an introduction to mathematical models. London: Imperial College Press; 2010.

[40] Ortigueira M, Machado JAT, Trujillo J, Vinagre B. Advances in fractional signals and systems. Signal Process 2011;91(3):349. doi:10.1016/j.sigpro.2010.08.002.

[41] Baleanu D, Diethelm K, Scalas E, Trujillo JJ. Fractional calculus: models and numerical methods, series on complexity, nonlinearity and chaos. Singapore: World Scientific Publishing Company; 2012.

[42] Ionescu C. The human respiratory system: an analysis of the interplay between anatomy, structure, breathing and fractal dynamics, series in bioengineering. London: Springer-Verlag; 2013.

[43] Machado JAT. Fractional generalization of memristor and higher order elements. Commun Nonlinear Sci Numer Simul 2013;18(12):264-75. doi:10.1016/j.cnsns.2012.07.014.

[44] Machado JAT. Fractional order generalized information. Entropy 2014;16(4):2350-61. doi:10.3390/e16042350.

[45] Hoare MR, Rahman M. On the spectral theory of Rayleigh's piston. I. The discrete spectrum. J Phys A: Math Nucl Gen 1973;6(10):1461-78. doi:10.1088/0305$4470 / 6 / 10 / 006$.

[46] Hoare MR, Rahman M. On the spectral theory of Rayleigh's piston. II. The exact singular solution for unit mass ratio. J Phys A: Math Nucl Gen 1974;7(9):107093. doi:10.1088/0305-4470/7/9/011.

[47] Hoare MR, Rahman M. On the spectral theory of Rayleigh's piston. III. Exact solution of the absorbing barrier problem ( $\gamma=1$ ). J Phys A: Math Nucl Gen 1976;9(1):77-85. doi:10.1088/0305-4470/9/1/013.

[48] Barker JA, Hoare MR, Raval S. A numerical study of the general Rayleigh's piston model. J Phys A: Math Gen 1981;14(2):423-38. doi:10.1088/03054470/14/2/018.

[49] Driessler W. On the spectrum of the Rayleigh piston. J Stat Phys 1981;24(4):595-606. doi:10.1007/BF01011377.

[50] Driessler W. On the spectrum of the Rayleigh piston II. J Stat Phys 1982;29(1):95-112. doi:10.1007/BF01008250.

[51] Gruber C, Frachebourg L. On the adiabatic properties of a stochastic adiabatic wall: evolution, stationary non-equilibrium, and equilibrium states. Phys A: Stat Mech Appl 1999;272(3-4):392-428. doi:10.1016/S0378-4371(99)00237-X.

[52] Garcia A, Meurs P, de Broeck CV. Rectification of thermal fluctuations in ideal gases. Phys Rev E 2004;70(5). doi:10.1103/PhysRevE.70.051109.

[53] Ehrhardt K, Ferré A, Villain-Guillot S, Würger A. Entropy production of a Rayleigh piston separating gases of different temperature. In: Proceedings of the 4th IEEE international conference on nonlinear science and complexity. Budapest; 2012. p. 15-18. doi:10.1109/NSC.2012.6304725.

[54] Sarracino A, Gnoli A, Puglisi A. Ratchet effect driven by Coulomb friction: the asymmetric Rayleigh piston. Phys Rev E 2013;87(4). doi:10.1103/PhysRevE.87.040101. 